\title{
Evaluación de la maloclusión, alteraciones funcionales y hábitos orales en una población escolar: Tarragona y Barcelona
}

\section{Evaluation of malocclusion, functional and oral habits alteration in a school student population: Tarragona and Barcelona}

\author{
García García VJ*, Ustrell Torrent JM**, Sentís Vilalta J***
}

\section{RESUMEN}

Fundamento: La etiología de la maloclusión puede ser multifactorial y difícil de clasificar durante el desarrollo del individuo. Los objetivos del estudio son conocer las características de la maloclusión y su posible relación con las alteraciones funcionales y los hábitos orales.

Métodos: Se estudió a 1.270 individuos representativos de la población escolar de Cataluña; 596 niños y 674 niñas de entre 6 y 14 años. Se obtuvo información mediante registros clínicos y cuestionarios. Se trata de un estudio observacional descriptivo mixto transversal, llevado a cabo entre 2006 y 2007 a través de un muestreo no probabilística de conveniencia. Se ha realizado el calibrado de los examinadores.

Resultados: Fueron incluidos 1051 escolares con una edad media de 9,32 años. El 72,8\% presenta según Angle Clase I, 19,0\% y 5,2\% Clase II/1 y II/2 respectivamente y 2,9\% Clase III. El hábito onicofagia fue el más frecuente con un 46,4\%. El mayor nivel de limitación funcional fue la hipertrofia amigdalar con un 21,2\%. Existe relación estadísticamente significativa entre maloclusión sagital y tipo de respiración y movilidad lingual y entre maloclusión horizontal y tiempo de succión digital y movilidad lingual $(p<0,05)$.

Conclusiones: El diagnóstico de alteraciones funcionales y de hábitos orales puede advertir de la presencia de maloclusión.

Palabras clave: Maloclusión, hábitos orales, alteración funcional, prevalencia, escolares.

\section{SUMMARY}

Background: Etiology of malocclusion may be multifactor and difficult to classify during the development of the individual. The aim of this study is to identify the malocclusion characteristics in a school student population and find the possible relation with functional and oral habit alteration.

Material and method: 1270 individuals representative of the school population of Catalonia were studied: 596 boys and 674 girls between 6 and 14 years. Information was obtained through medical records and questionnaires. A mixed observational-descriptive transversal study has been done in the period between 2006 and 2007 that was selected by a non-probabilistic convenience sampling. Interexamination calibration was preformed.

Results: 1051 schoolchildren were included in the study with a mean age of 9.32 years. $72.8 \%$ presented as Angle Class I, 19.0\% and 5.2\% Class II / 1 and II / 2, respectively and 2.9\% Class III. Nail biting habit was the most frequent habit with $46.4 \%$. The highest level of functional limitation was tonsils hypertrophy with $21.2 \%$. There is a statically significant relationship between sagittal malocclusion and respiration type and tongue mobility, and between horizontal malocclusion and digital suction and tongue mobility $(\mathrm{p}<0.05)$.

Conclusions: The diagnosis of functional disorders and oral habits can be a warning of the presence of malocclusion.

* Práctica privada de Ortodoncia en Tarragona.

** Profesor Titular de Ortodoncia. Departamento de Odontoestomatología. Universidad de Barcelona

*** Profesor Emérito. Departamento de Salud Pública. Universidad de Barcelona. 
Key words: Malocclusion, oral habits, functional alteration, prevalence, schoolchildren.

Fecha de recepción: 20 de diciembre de 2009.

Aceptado para publicación: 20 de enero de 2010.

García García VJ, Ustrell Torrent JM, Sentís Vilalta J. Evaluación de la maloclusión, alteraciones funcionales y hábitos orales en una población escolar: Tarragona y Barcelona. Av. Odontoestomatol 2011; 27 (2): 75-84.

\section{INTRODUCCIÓN}

La maloclusión es el resultado de la anormalidad morfológica y funcional de los componentes óseos, musculares y dentarios que conforman el sistema estomatognático. Los factores genéticos y el medio ambiente son los dos factores principales (1).

Según datos epidemiológicos, la tendencia en los países en desarrollo es la de aumentar la prevalencia de maloclusiones en su población general (2).Alrededor del $70 \%$ de la población infantil presenta algún grado de desviación de la oclusión ideal, y entre un 25 y un $30 \%$ necesita tratamiento ortodóncico. A su vez, las maloclusiones son factores de riesgo de otras afecciones orales tales como la gingivitis, la periodontitis, la caries y la disfunción en la articulación temporomandibular, a parte de producir alteraciones estéticas y funcionales, cosa que implica la necesidad de aplicar medidas preventivas adecuadas a cada momento (3).

En un estudio realizado en la Comunidad Autonómica Vasca en 1990, se determinó que había maloclusión en un 23,5\% a los 7 años de edad, un 27,6\% a los 12 años y un 26,6\% a los 14 años (4). Sin embargo, existen datos con prevalencias inferiores, como es el caso del estudio transversal realizado en escolares de Galicia en 1995, en el que se observa un 13,9\% de maloclusión moderada y un $16,5 \%$ de severa (5).

Otro factor de maloclusión es la alteración de la función oral (6). Ésta incluye la articulación, la deglución y la masticación que pueden ser afectadas por los hábitos orales y las alteraciones funcionales. Según Graber et al. la morfología y la función oral son inseparables como las ruedas de un vehículo (7). Las alteraciones funcionales y los hábitos orales pueden llegar a modificar la posición de los dientes, la relación de las arcadas dentarias entre si e interferir en el crecimiento normal y en la función. Un estudio epidemiológico realizado recientemente en España sobre una muestra de 1.100 escolares valoró que un 53,3\% presentaba algún hábito oral (8).Otro ejemplo de interés que plantea la relación entre hábitos y patología, es cuando ésta última se trata de la enfermedad periodontal. Aunque no se han encontrado evidencias clínicas de que los hábitos puedan ser factores etiológicos, si que es patente una posible relación (7).

La mayor parte de asistencia odontológica de la población se realiza a través del sistema privado y las prestaciones del sistema público se limitan todavía al diagnóstico y al alivio del dolor con carácter general de la población adulta, aunque para la población infantil adolescente, las prestaciones se han ampliado a programas preventivos (9). No obstante, en los planes de salud bucodental no se hace referencia a la necesidad de tratamiento de las alteraciones de la oclusión, cosa que podría hacer pensar que la ortodoncia está excluida de la planificación, prevención y asistencia en el ámbito del Sistema Nacional de Salud (10).

La escasez de estudios que caractericen de una manera más precisa las maloclusiones y valoren el papel que juega las alteraciones funcionales y los hábitos orales promueven la necesidad de actualizar los datos en nuestro país y de establecer un nuevo criterio de relación entre la patología y su etiología. Esto comporta no limitarse a definir si existe o no, es decir, se debe valorar que tipo de maloclusión existe y con que cursa. Debido a la mínima información de la que se dispone, se ha realizado un estudio piloto para enfocar las diferentes directrices de futuros trabajos de investigación. Así pues, nuestro objetivo es estudiar la maloclusión en una población escolar de Cataluña y su posible relación con las alteraciones 
funcionales y los hábitos orales. Como hipótesis nula se ha establecido que no existe diferencias estadísticamente significativas en la aparición de maloclusión en la población de 6 a 14 años, según exista o no hábito oral o alteración funcional.

\section{MATERIAL Y MÉTODOS}

El estudio se realizó en los CEIP (Centro de Enseñanza Infantil y Primaria) y en los IES (Instituto de Enseñanza Secundaria) que pertenecían a los C.A.P (Centros de Atención Primaria) de Tarragona y Barcelona de diciembre de 2006 a junio de 2007. Se trata de un estudio observacional descriptivo mixto transversal en el que se tuvo en cuenta consideraciones éticas, tales como el principio de beneficencia y de determinación. En cambio, el principio de justicia no se valoró al tratarse de un estudio observacional. Siendo la población diana individuos entre los 6 y los 14 años de edad, la población elegible fue los alumnos de primero, tercero y quinto de primaria de los CEIP y los de primero y tercero de ESO (Enseñanza Secundaria Obligatoria) de los IES. Como criterio de exclusión se estableció a los pacientes que habían recibido tratamiento de ortodoncia o que estuviesen en terapia y los síndromes que pudiesen afectar con alteraciones orofaciales. Se realizó un muestreo no probabilístico de conveniencia, siendo la fuente de información los datos clínicos de cada paciente y un cuestionario respondido por los padres o bien por los tutores legales.

Con el objetivo de asegurar una uniforme interpretación, comprensión y registro de los criterios de identificación de las variables a estudiar, y para minimizar la variabilidad entre los diferentes equipos examinadores, se procedió a la calibración en dos fases. ${ }^{11}$ En la primera fase se realizó una sesión teórica en donde se informó sobre el protocolo de estudio, sobre los criterios de diagnóstico, de tratamiento y sobre el modo de llevar a cabo la recogida de datos. En otra sesión teórico-práctica se aclararon las dudas con la ayuda de los pacientes de ortodoncia de la Clínica Odontológica de la Fundación Josep Finestres y que tenían las características de los individuos diana del estudio. En la segunda fase se realizaron las sesiones clínicas. El primer día se seleccionó aleatoriamente a los pacientes hasta llegar a un número de
15 que fueron estudiados individualmente por los examinadores. Después de 15 días se volvió a estudiar a los mismos pacientes de manera individual. Una vez recogidos los datos se procedió al análisis estadístico con la prueba de Mc Nemar que demostró la no existencia de diferencias significativas en las clasificaciones realizadas entre examinadores y con el Índice Kappa se comprobó que existía concordancia entre examinadores.

Como variable dependiente se analizó la maloclusión, que a su vez se clasificaba en problemas sagitales (Clase I, II/1, II/2 y III molar de Angle), problemas transversales (mordida cruzada, mordida en tijera, línea media desviada y normooclusión) y problemas verticales (sobremordida, mordida abierta y normooclusión). Como variable independiente se analizó las alteraciones funcionales de las narinas, de las amígdalas y de la movilidad lingual (12), con los valores que pueden observarse en la Tabla 1. La presencia de alteración en la respiración (nasal, oral y mixta), de succión digital (no, hasta los 2,5 años y más de 2,5 años), de onicofagia o el hábito de morder objetos (presencia o ausencia) fue detectada clínicamente y confirmada por los padres mediante un cuestionario diseñado para la ocasión. Éste último a su vez se empleó también para recoger datos sobre el uso del ejercitador.

Se utilizaron sets de exploración (sonda de exploración y espejo intraoral), las plantillas y un cuestionario con autorización de los padres o de los tutores.

El tamaño muestral se calculó en el supuesto de una variable dicotómica teniendo en cuenta que no se conocía resultados previos, se tomó el valor más desfavorable para el calculo del tamaño de la muestra, $p=q=0,5$, con una precisión del $4 \%$, puesto que se creía que era una amplitud adecuada y con un intervalo de confianza del 95\%. Así pues, el tamaño de la muestra mínimo calculo fue de 600 sujetos.

Para el análisis bivariante de la muestra se aplicó la prueba Ji al cuadrado de Pearson con un nivel de significación á del 0.05 y fue considerado estadísticamente significativo un valor 'p' menor de 0,05. Se utilizó el programa SPSS-PC 13.0 para Windows. Como limitaciones del estudio se aprecia que éste no fue aleatorio y las funciones como la respiración o la deglución pudieron quedar camufladas por otras 


\section{TABLA 1.- CODIFICACIÓN DE LAS ALTERACIONES SEGÚN DURÁN}

\section{Clasificación narina}

Tipo 0: narinas dilatadas tanto en reposo como en inspiración forzada.

Tipo 1: narinas estrechas o pequeñas en reposo pero sin colapso funcional.

Tipo 2: colapso parcial unilateral de las narinas.

Tipo 3: colapso funcional total unilateral o parcial bilateral.

Tipo 4: colapso funcional parcial de una narina y total en la otra.

Tipo 5: colapso funcional completo bilateral de las narinas.

\section{Clasificación amígdalas}

Tipo 0: se ha practicado amigdalectomía.

Tipo 1: no se ven, no invaden orofaringe (no intervenir).

Tipo 2: se ven moderadamente (no intervenir).

Tipo 3: ocupan 1/3 del espacio de la orofaringe (estar alerta).

Tipo 4: invaden 2/3 del espacio de la orofaringe (aconsejable intervenir $\mathrm{Qx}$ ).

Tipo 5: se tocan en la línea media ambas amígdalas

\section{Clasificación frenillo lingual}

Tipo 0: se ha practicado frenectomía.

Tipo 1: punta de la lengua toca el paladar.

Tipo 2: punta de la lengua casi toca el paladar.

Tipo 3: lengua situada en mitad de la boca (responde bien a la reeducación).

Tipo 4: punta lengua sobrepasa los incisivos inferiores (intentar reeducar).

Tipo 5: la punta de la lengua no sobrepasa los incisivos inf. (Cirugía).

patologías como pueden ser los procesos infecciosos. Tampoco se realizó una calibración intraestudio.

\section{RESULTADOS}

Se seleccionó una muestra de 1.270 escolares de los cuales se excluyeron 219 porque habían recibido tratamiento de ortodoncia o estaban en terapia y por tener algún síndrome que pudiese afectar con alteraciones orofaciales. Se han incluido 1051 individuos con una media de 9,32 años (DE 2,51), 496 niños $(47,2 \%)$ y 555 niñas $(52,8 \%)$. El $72,8 \%$ presenta según Angle Clase I, 19,0\% y 5,2\% Clase II/1 y II/2 respectivamente y 2,9\% Clase III. En la Tabla 2 se puede apreciar la distribución de la maloclusión vertical y horizontal. Se ha observado que el $46,4 \%$ de la población estudiada presenta el hábito onicofagia o bien de morder objetos. Del total de los escolares el 15,0\% presenta respiración oral, el 70,6\% respiración nasal y el $14,4 \%$ ambos tipos de respiración. En la Figura 1 se puede valorar la distribución de hábitos como la

\begin{tabular}{|l|r|r|}
\hline \multicolumn{3}{|c|}{ TABLA 2.- DISTRIBUCIÓN DE LA } \\
MALOCLUSIÓN HORIZONTAL Y \\
VERTICAL \\
\hline Maloclusión horizontal & n & $\%$ \\
\hline - Mordida cruzada & & \\
\hline - Mordida en tijera & 69 & 6,5 \\
\hline - Línea media desviada & 5 & 0,5 \\
\hline - Normooclusión & 195 & 18,6 \\
\hline - Mord. cruzada /línea desviada & 656 & 62,4 \\
\hline Maloclusión vertical & 126 & 12 \\
\hline - Sobremordida & & \\
\hline - Mordida abierta & 353 & 33,6 \\
\hline - Normooclusión & 121 & 11,5 \\
\hline
\end{tabular}

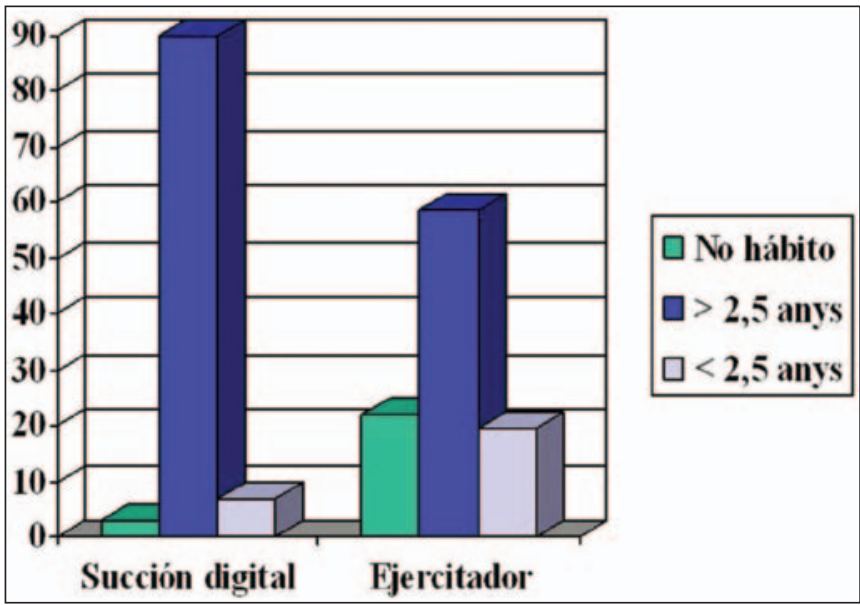

Fig. 1. Porcentajes de las variables succión digital y uso del según la duración. 


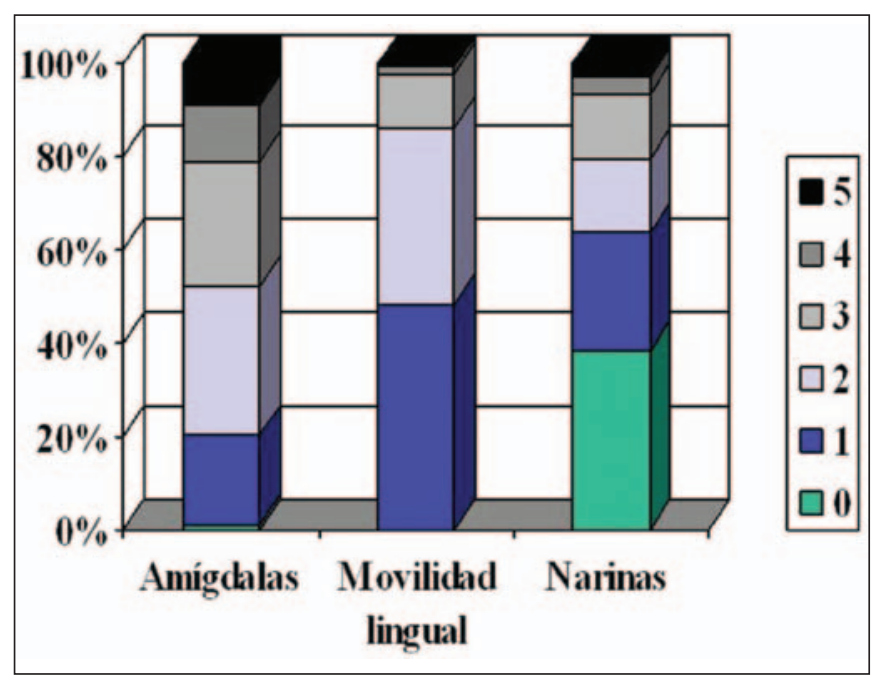

Fig. 2. Porcentajes de las variables amígdalas, movilidad lingual y narinas según su tipología. succión digital y el uso del ejercitador en función de la duración, mientras que en la Figura 2 se presenta la distribución de las alteraciones funcionales en relación a los valores establecidos para el estudio.

En la Tabla 3 se especifican los porcentajes obtenidos al analizar la relación entre tipo de maloclusión y los hábitos orales que pueden originarlos. Se ha establecido relación entre alteración horizontal y succión digital con un valor $\chi^{2}$ de 19,7, con 8 gl y una significación de $p<0,02$, y se han calculado los residuos corregidos para buscar entre que casillas existe relación. Se ha encontrado entre succión digital $>2,5$ años y normooclusión $\mathrm{p}<0,02$ y entre succión digital $>2,5$ años y maloclusión horizontal mordida cruzada y línea media desviada $p<0,02$. También se ha hallado relación entre alteración sagital y respiración con

\section{TABLA 3.- DISTRIBUCIÓN DE LA MALOCLUSIÓN EN RELACIÓN A LOS HÁBITOS ORALES}

\begin{tabular}{|c|c|c|c|c|c|c|c|c|}
\hline & \multicolumn{2}{|c|}{$\begin{array}{l}\text { Ejercitador } \\
>2,5 \text { años }\end{array}$} & \multicolumn{2}{|c|}{$\begin{array}{c}\text { Succión digital } \\
2,5 \text { años }\end{array}$} & \multicolumn{2}{|c|}{$\begin{array}{c}\text { Respiración } \\
\text { oral }\end{array}$} & \multicolumn{2}{|c|}{ Onicofagia } \\
\hline & n & $\%$ & n & $\%$ & n & $\%$ & n & $\%$ \\
\hline \multicolumn{9}{|l|}{ Maloclusión sagital } \\
\hline - Clase I Angle & 144 & 70,6 & 53 & 71,6 & 113 & 71,5 & 367 & 75,7 \\
\hline - Clase II/1 Angle & 44 & 21,5 & 17 & 23,0 & 27 & 17,1 & 87 & 18,0 \\
\hline - Clase II/2 Angle & 11 & 5,4 & 1 & 1,4 & 7 & 4,4 & 18 & 3,7 \\
\hline - Clase III Angle & 5 & 2,5 & 3 & 4,0 & $11^{* *}$ & 7,0 & 13 & 2,6 \\
\hline \multicolumn{9}{|l|}{ Maloclusión horizontal } \\
\hline - Mordida cruzada & 13 & 6,4 & 8 & 10,9 & 5 & 3,1 & 33 & 6,8 \\
\hline - Mordida en tijera & 2 & 1,0 & 0 & 0,0 & 0 & 0,0 & 2 & 0,4 \\
\hline - Línea media desviada & 42 & 20,6 & 8 & 10,9 & 34 & 21,5 & 95 & 19,6 \\
\hline - Normooclusión & 123 & 60,2 & $56^{*}$ & 75,6 & 96 & 60,8 & 305 & 62,9 \\
\hline - Mor. cruzada/línea desviada & 24 & 11,8 & $2^{*}$ & 2,6 & 23 & 14,6 & 50 & 10,3 \\
\hline \multicolumn{9}{|l|}{ Maloclusión vertical } \\
\hline - Sobremordida & 74 & 36,3 & 21 & 28,3 & 49 & 31,0 & 163 & 33,6 \\
\hline - Mordida abierta & 21 & 10,3 & 9 & 12,2 & 27 & 17,1 & 54 & 11,1 \\
\hline - Normooclusión & 109 & 53,4 & 44 & 59,5 & 82 & 51,9 & 268 & 55,3 \\
\hline
\end{tabular}

Diferencias significativas con $* p<0,02, * * p=0,03$. 
una $\chi^{2}$ de 13,98, con 6 gl y significación $p=0,03$ estableciéndose entre maloclusión sagital de Clase III y respiración oral $p=0,03$. Se puede apreciar la elevada prevalencia de pacientes con línea media desviada $(36,1 \%)$ que presentan respiración oral y que los pacientes del estudio que tienen hábitos inadecuados cursan con altas porcentajes de maloclusión vertical (ejercitador $>2,5$ años 46,6\%, succión digital $>2,5$ años $40,5 \%$, respiración oral $48,1 \%$ y onicofagia $44,7 \%$ ).

En la Tabla 4 se establece la relación proporcional entre las variables maloclusión y los valores más altos de las limitaciones funcionales. En ésta última se aprecia que existe una relación significativa entre las variables maloclusión sagital y movilidad lingual con un valor de $\chi^{2}$ de 27,3, con 9 gl y significación $p<0,01$ existiendo relación entre maloclusión sagital de Clase I de Angle y movilidad lingual valor $3 p<0,01$ y maloclusión sagital Clase I de Angle y valor $4 \mathrm{p}<0,01$, habiéndose calculado los residuos corregidos. En la misma tabla se halla relación entre maloclusión horizontal y movilidad lingual con una $\chi^{2}$ de 23,5 , con $12 \mathrm{gl}$ y significación $\mathrm{p}<0,03$. Está se puede apreciar entre maloclusión horizontal normooclusión y movilidad lingual tipo $3 \mathrm{p}<0,03$ y maloclusión horizontal mordida cruzada y línea media desviada y movilidad lingual tipo $3 p<0,03$. Cabe destacar la alta prevalencia que se establece entre narinas valor 5 y maloclusión vertical sobremordida.

\section{DISCUSIÓN}

El hecho de clasificar la maloclusión en ligera, moderada y severa tal y como indica la OMS, limita una caracterización precisa de la patología que puede

\begin{tabular}{|c|c|c|c|c|c|c|c|c|c|c|c|c|}
\hline \multicolumn{13}{|c|}{$\begin{array}{l}\text { TABLA 4.- DISTRIBUCIÓN DE LA MALOCLUSIÓN EN RELACIÓN A LOS VALORES CON } \\
\text { MÁS LIMITACIÓN FUNCIONAL }\end{array}$} \\
\hline & \multicolumn{4}{|c|}{ Hipertrofia amigdalar } & \multicolumn{4}{|c|}{ Movilidad lingual } & \multicolumn{4}{|c|}{ Narinas } \\
\hline & \multicolumn{2}{|c|}{ Valor 4} & \multicolumn{2}{|c|}{ Valor 5} & \multicolumn{2}{|c|}{ Valor 3} & \multicolumn{2}{|c|}{ Valor 4} & \multicolumn{2}{|c|}{ Valor 4} & \multicolumn{2}{|c|}{ Valor 5} \\
\hline & $\mathrm{n}$ & $\%$ & $\mathrm{n}$ & $\%$ & $\mathrm{n}$ & $\%$ & $\mathrm{n}$ & $\%$ & $\mathrm{n}$ & $\%$ & $\mathrm{n}$ & $\%$ \\
\hline $\begin{array}{l}\text { Maloclusión sagital } \\
\text { - Clase I Angle }\end{array}$ & 87 & 66,4 & 64 & 70,3 & $80^{*}$ & 70,2 & $9^{*}$ & 45 & 30 & 76,9 & 17 & 58,6 \\
\hline - Clase II/1 Angle & 33 & 25,2 & 19 & 20,9 & 26 & 22,8 & 7 & 35 & 8 & 20,5 & 9 & 31 \\
\hline - Clase II/2 Angle & 7 & 5,3 & 3 & 3,3 & 8 & 7 & 1 & 5 & 1 & 2,6 & 1 & 3,5 \\
\hline - Clase III Angle & 4 & 3,1 & 5 & 5,5 & 0 & 0 & 3* & 15 & 0 & 0 & 2 & 6,9 \\
\hline \multicolumn{13}{|l|}{ Maloclusión horizontal } \\
\hline - Mordida cruzada & 8 & 6,1 & 9 & 9,9 & 7 & 5,7 & 1 & 5 & 3 & 7,7 & 5 & 16,1 \\
\hline - Mordida en tijera & 0 & 0 & 0 & 0 & 1 & 0,9 & 0 & 0 & 0 & 0 & 0 & 0 \\
\hline - Linea media desviada & 19 & 14,5 & 15 & 16,5 & 25 & 20,3 & 4 & 20 & 10 & 25,6 & 5 & 16,1 \\
\hline - Normooclusión & 85 & 64,9 & 58 & 63,7 & $64^{* *}$ & 52 & 13 & 65 & 22 & 56,4 & 19 & 61,3 \\
\hline - M.cruzada/lineadesviada & 19 & 14,5 & 9 & 9,9 & $26^{* *}$ & 21,1 & 2 & 10 & 4 & 10,3 & 2 & 6,5 \\
\hline \multicolumn{13}{|l|}{ Maloclusión vertical } \\
\hline - Sobremordida & 52 & 39,7 & 31 & 34 & 45 & 36,6 & 7 & 35 & 12 & 30,8 & 18 & 62,1 \\
\hline - Mordida abierta & 17 & 13 & 17 & 18,7 & 7 & 5,7 & 1 & 5 & 4 & 10,2 & 2 & 6,9 \\
\hline - Normooclusión & 62 & 47,3 & 43 & 47,3 & 71 & 57,7 & 12 & 60 & 23 & 59 & 9 & 31 \\
\hline
\end{tabular}

Diferencias significativas con $* p<0,01,{ }^{* *} p=0,03$. 
presentar el niño (13). Los datos obtenidos de la alteración sagital en este estudio se encuentran dentro de los parámetros normales coincidiendo con los registros de Onyeaso con un $74,5 \%$ de Clase I, un $13,7 \%$ de Clase II y un 11,8 de Clase III (14). Son valores similares a los de Montiel con un $68 \%$ de Clase I, un 32 de Clase II y un 9 de Clase III (15).

Al analizar la alteración horizontal, se puede apreciar que la desviación de la línea media fue de un $25 \%$ en la muestra de Montiel y que concuerda con el 30,5\% que se ha encontrado en este estudio. Por otro lado, encontramos frecuencias que contrastan con los resultados hallados como es el 3,1\% a la edad de 6 años y el $6,7 \%$ a los 12 años en poblaciones escolares de Galicia (5).

Se aprecia la elevada proporción de mordida cruzada posterior que se registró $(18,6 \%)$ y que concuerda con los datos de referencia que documentan otros autores (16). Sin embargo, existen otros estudios que revelan proporciones menores (4\%) (17). Por lo tanto, una rápida intervención ortodóncica, que se consigue mediante la ortodoncia interceptiva, puede ayudar a un normal desarrollo de la oclusión y de la armonía facial y evitar así el empeoramiento de la maloclusión (18).

\section{Hábitos orales-maloclusión}

\section{Onicofagia-Morder objetos}

Es el hábito oral más frecuente de la muestra tratándose de una proporción muy superior si se compara con estudios realizados por Bosnjak et al. (28\%) (19) o por Montiel (34\%) (15). Además, en este último estudio se encontraron diferencias estadísticamente significativas en el género, siendo superior la frecuencia en el sexo femenino. Según éste, la etiología de esta desproporción sería la diferente educación sexista recibida entre ambos sexos (15).

Datos de investigaciones anteriores proponen que este hábito pernicioso es el resultado del nerviosismo habitual en los niños, de su incertidumbre y de su inquietud (20-22). El tratamiento de este problema se debe afrontar con un tratamiento multidisciplinario mediante la combinación de procedimientos psicológicos, pediátricos y odontológicos (23).

\section{Succión digital}

La succión digital puede aparecer como consecuencia de ciertas conductas regresivas frente a algunos trastornos emocionales, asociados a la inseguridad o al deseo de llamar la atención. En la muestra estudiada, al tratarse de grupos de edades diferentes, se valoró el uso y la duración, de manera que un $10,1 \%$ se había succionado el dedo y de estos el 69,1\% lo hicieron como mínimo hasta los 2,5 años.

Un punto importante a la hora de interpretar los datos inferenciales entre este hábito y la patología mordida cruzada posterior, es que los pacientes que se habían succionado el dedo durante más de 2,5 años representaban el 5,1\% de la patología mientras que los que no lo habían hecho representaban el 1,5\% existiendo relación estadísticamente significativa.

\section{Ejercitador}

El ejercitador puede producir efectos similares a los de la succión digital y por lo tanto debe ser suprimido en el momento en que se produce la erupción de los incisivos temporales, si queremos evitar la maloclusión (24). No obstante, cuando se suprime el hábito, es frecuente la autocorrección incluso si el hábito se prolonga hasta los 4 años de edad (23).

Cuando el uso persiste, se altera la posición de los dientes y aparece una mordida abierta anterior, como consecuencia del efecto físico que se genera sobre la erupción normal de los dientes, además de producir compresión y protusión del maxilar (5).

En esta muestra se aprecia que el $78 \%$ ha utilizado el chupete y aunque no se han encontrado datos estadísticamente significativos, si se observa que los pacientes que lo usaron durante más de 2,5 años representan el 18,9\% del total de la patología mordida cruzada posterior.

El papel que desempeñan los profesionales sanitarios, que reconocen a los niños en edades muy jóvenes, al advertir sobre la posibilidad de efectos secundarios en la boca por el uso prolongado del 
ejercitador, es importante para hacer disminuir esa alta frecuencia de patología asociada al hábito.

\section{Respiración oral}

La frecuencia del $15 \%$ de respiradores orales valorada en los escolares es comparable a la detectada por Montiel en su muestra (16\%) (15).

La posición baja que adopta la lengua para que pueda circular el aire por la boca produce una serie de cambios como son la apertura bucal permanente por hipotonicidad labial, un crecimiento transversal mandibular, freno del crecimiento transversal maxilar y elevación de la bóveda palatina (paladar ojival), protusión de los incisivos, debilidad de la musculatura facial con aumento del tercio inferior de la cara, retrusión del mentón, posterorrotación mandibular y labio superior corto e inferior grueso. Todos estos cambios comportan el aspecto o síndrome de cara larga o facies adenoidea (25). En este estudio se ha encontrado un $25,4 \%$ de mordida cruzada posterior, siendo una prevalencia elevada pero que no es estadísticamente significativa.

Del total de la población estudiada se ha observado un 2,6\% de niños cursaban con maloclusión sagital Clase II/1 según Angle y con respiración oral. Sin embargo, en esta muestra se ha podido apreciar que existe una relación estadísticamente significativa entre los respiradores orales y la maloclusión sagital de Clase III con una $\mathrm{p}<0,02$. El hecho de que un $36,7 \%$ de la población con Clase III curse con este hábito indica una observación más detenida de estos pacientes.

\section{Alteraciones funcionales-maloclusión}

\section{Narinas}

Con la codificación del funcionalismo de las narinas se puede valorar el grado de colapso durante la inspiración forzada (12). El valor 4 presenta un colapso funcional parcial de una narina y total de la otra, mientras que el valor 5 expresa un colapso funcional total en ambas narinas. Según Durán, estos tipos de grados de alteración funcional pueden forzar a una respiración oral y comporta una serie de efectos. En la muestra estudiada se presenta este grado de colapso en un 6,5\% reflejando una baja frecuencia y no se ha encontrado relación estadísticamente con ningún tipo de maloclusión. No obstante, este colapso grave presente en la población escolar debería ser estudiado y valorado con la finalidad de diagnosticar el factor etiológico, de manera que una derivación al otorrinolaringólogo estaría indicada.

\section{Hipertrofia amigdalar}

En cuanto al grado de hipertrofia amigdalar de los individuos de la muestra se aprecia que el 20,2\% presentaban un valor entre 4 y 5 (amígdalas que ocupan dos terceras partes del espacio de la orofaringe y amígdalas que ocupan la totalidad del espacio y contactan entre sí respectivamente). Esta hipertrofia comportaría la indicación de intervención quirúrgica según las recomendaciones de Durán. La alteración morfológica, independientemente de su etiología, puede producir una disfunción respiratoria entre otras, en este caso representada por la respiración bucal (12). Además, el agrandamiento de las amígdalas palatinas actúa como factor inhibidor del crecimiento normal y es causante de una interposición lingual que puede crear mordida abierta anterior (26).

Como se a comentado anteriormente, esta respiración bucal puede producir posterorrotación, aunque si ésta es incipiente, se trataría de una situación postural. Ahora bien, si se consigue en este estadio rehabilitar la disfunción respiratoria, se puede restablecer el patrón muscular y eruptivo. Esto provocaría una progresiva anterorrotación mandibular y en consecuencia el restablecimiento de la normalidad esquelética (27). Apoyando esta filosofía de tratamiento se encuentran los estudios realizados por McNamara, en los cuáles se demostró que después de realizar una adenoidectomía, se producía la comentada anterorrotación mandibular, ya que se normalizaba la respiración, la deglución y la masticación (28).

\section{Movilidad lingual}

En el caso de una movilidad lingual de valor 4 y 5 (la punta de la lengua sobrepasa ligeramente los incisi- 
vos inferiores y la punta de la lengua no sobrepasa a los incisivos inferiores respectivamente), se produce un movimiento de protección durante la deglución (12). La frecuencia de esta limitación funcional representa únicamente el 1,9\% de la muestra y por lo tanto una baja proporción de indicación para la intervención quirúrgica. Aun así, a éste porcentaje se debería sumar el $11,7 \%$ de los escolares que presentan un valor 3 , en donde la punta de la lengua llega a la mitad de la distancia entre los incisivos superiores e inferiores con la boca abierta. Con este grado de limitación estaría indicada la rehabilitación funcional y no cabe decir el papel importante que juega la figura del logopeda. También cabe destacar que después de una intervención quirúrgica de frenillo lingual es imprescindible el tratamiento de logopedia. Por lo tanto, es indiscutible que casos con sólo un tipo de limitación funcional, como es el caso, propongan un tratamiento multidisciplinar en el que actúen la figura del logopeda, del cirujano oral o maxilofacial y del ortodoncista. Además, si ya de por si son importantes las alteraciones que se producen en la deglución, se les debe añadir los problemas ortopédicos y ortodóncicos derivados. La limitación de la función de la lengua, expresada como una posición baja, se manifiesta con diversas patologías. Una de éstas, es la inhibición de la expansión del paladar, es decir, una mordida cruzada posterior. Prueba de ello es la relación estadísticamente significativa que se ha hallado en los escolares de la muestra. Otra patología que puede producir el frenillo labial corto se manifiesta con la mordida cruzada anterior. Esta posición baja facilita una posición avanzada de la mandíbula y el desarrollo del resalte negativo (9). Estas afirmaciones se corresponden con la relación estadísticamente significativa hallada entre maloclusión sagital dental de Clase III y movilidad lingual.

\section{AGRADECIMIENTOS}

Este trabajo ha sido posible gracias a la atención de la Sra. E. Grifell y del Sr. J. Hierro del centro corporativo del ICS (Institut Català de la Salut) y a la ayuda en la recogida de datos del Dr. R. Solé de la Unidad de Salud Bucodental del ICS de Tarragona y de la Dra. S. Colomer del departamento de Ortodoncia de la Universidad de Barcelona

\section{BIBLIOGRAFÍA}

1. Harris JE, Kowalski CJ. All in the family: Use of familial information in orthodontic diagnosis, case assessment, and treatment planning. Am J Orthod Dentofac Orthop. 1976;69:493-510.

2. Weiland FJ, Jonke E, Bantleon HP. Secular trends in malocclusion in Austrian men. Eur $\mathrm{J}$ Orthod. 1997;19:355-9.

3. Machuca C, Martínez F. Tratamiento de las mordidas cruzadas posteriores con Quad Hélix. Ortod Esp 1998;38:174-8.

4. Aubrey Sheiham (1995) Estudio epidemiológico de la salud buco-dental infantil en la comunidad Autónoma Vasca. Serie de documentos técnicos de salud publica dental. Vitoria Editorial: Servicio Central de Publicaciones, Gobierno Vasco. 115 .

5. Lorenzo V, Smyth E, Hervada X, Fernández R, Alonso JM, Amigo M, González-Zaera J, Montes A, Taracido M, Cerdá T. La salud bucodental en los escolares gallegos. 1995. Rev Esp Salud Pública 1998;72:539-46.

6. Camps D, Sánchez M, Ustrell JM. Conceptos básicos de ortodoncia (II). Indicaciones del tratamiento de la maloclusión dental. FMC 2001; 8:15-23.

7. Graber TM, Chung DDB, Aoba JT. Dentofacial orthopedics versus orthodontics. J Am Dent Assoc 1967;75:1145-66.

8. Paredes V, Paredes C. Prevalencia de los hábitos bucales y alteraciones dentarias en escolares valencianos. An Pediatr 2005;62:261-5.

9. Martinicorena JC, Llodra JC. Capt. 14 Salud pública bucodental. En: Juan M. Cabasés, Joan R. Villalbí, Carlos Aibar. Invertir para la salud. Prioridades en salud pública .Informes Sespas: Madrid; 2002.

10. Pérez F. Prevalencia de la maloclusión en una población escolar de la comunidad de les Illes 
Balears [Tesis Doctoral]. Universitat de Barcelona; 1995.

11. Rubio JM, Robledo T, Llodra JC, Simón F, Artazcoz J, González VL, García-Camba JM. Criterios mínimos de los estudios epidemiológicos de salud dental en escolares. Rev Esp Salud Pública. 1997;71:231-42

12. Durán J, Ustrell. Técnica MFS. Diagnóstico de la matriz funcional: codificación. Ortod clin. 2003;6: $138-40$

13. WHO. ORAL HEALTH SURVEYS: BASIC METHODS. $3^{\text {rd }}$ ed. Geneva: WHO; 1984.

14. Onyeaso CO. Prevalence of malocclusion among adolescents in Ibadan, Nigeria. Am J Orthod Dentofac Orthop 2004;126:604-7.

15. Montiel ME. Frecuencia de maloclusiones y su asociación con hábitos perniciosos en una población de niños mexicanos de 6 a 12 años de edad. ADM. 2004;61:209-14.

16. Ustrell JM, Duran J. Ortodoncia. Textos docents. Barcelona: Edicions de la Universitat de Barcelona; Barcelona; 243.

17. Duran Von Arx J. Problemas odontológicos en el niño. En: Callabed J, Moraga F, Sasot J, editores. La salud en la escuela y la guardería. Aspectos biológicos, psicológicos y sociales. Barcelona: Alertes; 1995.

18. Freeman JO. Preventive and interceptive orthodontics: a critical review and the results of a clinical study. J Prev Dent 1997;4:7-23.

19. Bosnjak A, Vucicevic-Boras V, Miletic I, Bozic Darko, Vukelja M. Incidence of oral habits in children with mixed dentition. J Oral Rehabil 2002;29:902-5.

20. Kawata T, Nakagawa H, Ohtani N, Kushima F, Ishida $\mathrm{N}$. Experimental study of abnormal habits and malocclusion - acquirement of finger habits. Nippon Kyosei Shika Gakkai Zasshi 1971:30;1823.
21. Kontogoures G. (1972) Harmful oral habits of children from the viewpoint of pediatric psychiatry. Hellenic Stomatol Chronicle 1972:16; 193.

22. Morban F. Oral habits in children and their psychological trauma. Acta Odontol Pediátr 1982:3;6-15.

23. Subtelny JD, Subtelny JD. Oral habits-Studies in form, function and therapy. Angle Orthod 1973; 43:347-83.

24. Camps D, Sánchez M, Ustrell JM. Conceptos básicos de ortodoncia (II). Indicaciones del tratamiento de la maloclusión dental FMC 2001;8:1523.

25. Camps D, Forés A, Duran J. Conceptos básicos de ortodoncia (I). Valoración de la oclusión. FMC 2000:7;653-61.

26. Christensen J, Fields H. Hábitos bucales. En: Pinckham JR, Cassamino P, Fields M, Mc Tigue $\mathrm{D}$, Nowak A, editores. Odontología pediátrica $\left(2^{\mathrm{a}}\right.$ ed.). México: Interamericana McGraw-Hill 1996; 375-82.

27. Durán J. Interrelación entre crecimiento máxilofacial y el síndrome de obstrucción respiratoria: a propósito de un caso. Rev Esp Ortod 1983;8:17.

28. McNamara J Jr. Influence on respiratory pattern on craniofacial growth. Angle Orthod. 1981;51: 296-300.

\section{CORRESPONDENCIA}

Valentín Javier García García

Facultad de Odontolgía.

Departamento de Ortodoncia. Despacho 2.7

Feixa Llarga, s/n

Hospitales de Llobregat.

Barcelona.

Correo electrónico: valentingarcia2@gmail.com 